'Departamento de Farmacología,

Facultad de Ciencias Biológicas, Universidad de Concepción, Concepción, Chile.

${ }^{2}$ Departamento de Psiquiatría y Salud Mental, Facultad de Medicina, Universidad de Concepción, Concepción, Chile. ${ }^{a} Q u i ́ m i c o$ Farmacéutico bPsicólogo (a). 'Bióloga.

${ }^{d}$ Doctorando en Salud Mental. eDoctor en Ciencias (PhD).

Estudio financiado por el Proyecto FONDECYT No1110687.

Recibido el 6 de junio de 2014, aceptado el 22 de julio de 2015.

Correspondencia a: Romina Rojas P, PhD. Departamento de Farmacología Facultad de Ciencias Biológicas Universidad de Concepción. Fono: +56-412661192 romrojas@udec.cl

\section{Asociación entre los polimorfismos 5HTTLPR, uMAOA y depresión en una cohorte de pacientes de atención primaria}

\author{
ROMINA ROJAS ${ }^{1, \mathrm{a}, \mathrm{e}}$, BENJAMÍN VICENTE $^{2, \mathrm{e}}$, SANDRA SALDIVIA $^{2, \mathrm{~b}, \mathrm{e}}$, \\ ROBERTO MELIPILLÁN ${ }^{2, b}$, GERALDINE AEDO ${ }^{1, \mathrm{c}}$, \\ NAÍN HORMAZABAL ${ }^{2, \mathrm{~d}}$, ANA CARROZA ${ }^{2}$
}

\section{Association between serotonin transporter and monoamine oxidase A gene polymorphisms and depression}

Background: Serotonin plays a central role regulating mood and on the development of depressive disorders. Aim: To study whether 5HTTLPR functional polymorphisms in the serotonin transporter gene or the Monoamine oxidase A gene (uMAOA) were risk markers for depression. Material and Methods: The Composite International Diagnostic Interview (CIDI) was applied to 1,062 consultants in primary health care centers aged between 18 and 75 years to establish the diagnosis of depression. A sample of saliva was obtained for DNA extraction and genetic analyses. Results: No association between the presence of depressive disorders and 5HTTLPR (ss) or uMAOA (3/3) risk genotypes was found. Psychological abuse and the presence of two or more life events were found to be predictors of depression in the studied sample. Conclusions: In this study, 5HTTLPR and UMAOA polymorphisms were not risk factors for depression. However, psychological abuse and the presence of two or more life events were risk factors for depressive disorders.

(Rev Med Chile 2015; 143: 1252-1259)

Key words: Depression; Polymorphism, genetic; Serotonin.
L os estudios familiares en el ámbito de la genética cuantitativa han demostrado que la depresión es un trastorno que presenta agregación familiar, con tasas de heredabilidad estimada en torno a $40 \%{ }^{1,2}$. Los estudios de gemelos y de adopción también apoyan la existencia de un componente hereditario o genético en la etiología de la depresión ${ }^{3,4}$.

Sin embargo, a pesar de los avances de la genética molecular en los últimos años, la base molecular de la herencia aún no se ha aclarado. La depresión se cree que es el resultado de la compleja interacción entre los factores genéticos y no genéticos, cada uno haciendo una pequeña contribución al fenotipo final ${ }^{5}$. En las últimas décadas, se han propuesto diferentes genes de riesgo para la depresión, establecidos en base a mutaciones o polimorfismos de riesgo que presentan en su secuencia génica, pero los resultados son generalmente poco concluyentes. Entre los hallazgos más replicados está la asociación descrita entre la variabilidad en la región promotora en el gen del transportador de serotonina (SERT) y un mayor riesgo para la ansiedad y la depresión ${ }^{6-9}$. El transportador de serotonina (SLC6A4) es un gen candidato para la depresión, ya que desempeña un 
papel clave en la neurotransmisión serotoninérgica y su producto proteíco es el blanco terapéutico para la mayoría de los fármacos antidepresivos. Uno de los polimorfismos descritos en este gen es el 5-HTTLPR, consiste en una inserción/deleción de $43 \mathrm{pb}$ en la región del promotor del gen ${ }^{10-12}$. Su variante corta (alelo s) estaría asociado a la disminución de la eficiencia de la transcripción del gen, resultando en una disminución de la expresión del transportador de serotonina en la neurona ${ }^{8}$. Estudios independientes han demostrado que los individuos con alelos s tienden a tener una peor respuesta al tratamiento antidepresivo con inhibidores selectivos de la recaptación de serotonina (ISRS) ${ }^{13-15}$. Sin embargo, la suposición que la expresión de variantes se asocia con trastornos mentales o la respuesta a los antidepresivos ha sido cuestionada ${ }^{16,17}$. En consecuencia, el papel de los genotipos del 5HTTLPR en el origen de la depresión permanece aún desconocido.

En una revisión, López- León y cols. ${ }^{9}$ analizaron todos los estudios de asociación genética en depresión mayor llevados a cabo hasta ese momento y la conclusión fue que de todos los genes que se habían explorado, evidencia de implicación estadísticamente significativa se encontró en sólo seis: APOE, DRD4, GNB3, MTHFR, SLC6A3 (DAT1), y SLC6A4 (SERT), a estos se podrían sumar otros genes como MAOA y BDNF que también han mostrado una tendencia a la asociación'. El gen de la monoamina oxidasa A (MAOA) también está involucrado en la vía serotoninérgica, y contiene una región dentro de su promotor que es polimórfica con respecto al número de copias de una repetición de 30 pares de base. Los alelos que han sido identificados para el polimorfismo de variación en número de repeticiones en tándem (MAOA-uVNTR) incluye los alelos con 3, 3,5, 4, y 5 repeticiones $(3 \mathrm{R}, 3.5 \mathrm{R}, 4 \mathrm{R}, \mathrm{y} 5 \mathrm{R})^{18}$. Estas diferentes variantes uVNTR se asocian con diferentes actividades transcripcionales del promotor $M A O A^{19}$, que a su vez da lugar a diferentes niveles de expresión del gen. El alelo del polimorfismo $M A O A$ uVNTR que resulta en altos niveles de expresión (alelo relacionado con alta actividad) ha sido asociado con suicidio causado por depresión en hombres ${ }^{20}$. Así también, el equipo de investigación PREDICT- GENE ha demostrado la existencia de un asociación significativa entre las variantes específicas de los genes SERT, MAOA y depresión $^{6,21}$. En estos últimos trabajos existe una fuerte asociación con los diferentes fenotipos de gravedad clínica, la que permanece aún después de ajustar los análisis debido al efecto de posibles factores de confusión.

El objetivo del presente trabajo es identificar la asociación entre los genotipos de riesgo de las variantes 5HTTLPR y uMAO en los genes SLC6A4 y $M A O A$ y la incidencia de depresión detectada en una muestra aleatoria de consultantes de centros de atención primaria de la Provincia de Concepción, Chile.

\section{Material y Método}

\section{Muestra y diseño del estudio}

Estudio de cohorte prospectivo, realizado sobre una muestra de pacientes atendidos en 10 centros de atención primaria de la Provincia de Concepción, Chile. Entre los años 2005 y 2008, el estudio PREDICT-FONDEF tuvo por objeto desarrollar un algoritmo predictivo de depresión en base a factores psicosociales en una cohorte de pacientes de atención primaria ${ }^{39}$. En el presente estudio, de los 2.832 pacientes del estudio original $2.466(87,1 \%)$ fueron exitosamente contactados en sus domicilios entre abril y octubre del año 2012, lo que corresponde al Tiempo 0 (T0). Se excluyeron a aquellos que ya tenían diagnóstico de depresión con el objeto de determinar la incidencia de patología depresiva en el seguimiento de éstos a los 12 meses (año 2013). En la primera entrevista se solicitó la participación de los pacientes, expresada a través de la firma de un formulario de consentimiento informado. Entrevistadores previamente entrenados recogieron la información y tomaron una muestra de saliva para el análisis genético. La recolección de datos de las variables ambientales y del uso de servicios se realizó en la primera medición, la recolección de datos para un eventual diagnóstico de trastorno depresivo (TD), trastorno por estrés postraumático (TEPT) y trastorno por ansiedad generalizada (TAG) (Composite International Diagnostic Interview, CIDI), se realizó a los 12 meses, entre abril y octubre del 2013 (Tiempo 1, T1). Los criterios de exclusión para todos los centros incluyeron la inhabilidad para entender el idioma local, la presencia de psicosis, demencia y/o de una enfermedad física incapacitante. Cada persona que aceptó participar firmó un formulario de consentimiento informa- 
do y los protocolos del estudio fueron aprobados y visados por el Comité de Ética de la Facultad de Medicina de la Universidad de Concepción. Inicialmente, el número de participantes de los cuales se disponía muestra genética fue 1.602. Sin embargo, debido a la presencia de valores ausentes en alguno de los predictores incluidos en los análisis, la muestra final evaluada fue de 1.223 participantes. La mayoría de los sujetos que recibieron algún diagnóstico psiquiátrico CIDI (TD, TEPT o TAG) se encontraban en tratamiento en el CESFAM o nivel secundario respectivo, a los restantes se les notificó por carta certificada el hallazgo, sugiriendo alternativas de atención.

\section{Extracción de ADN}

El ADN genómico fue aislado desde $1 \mathrm{ml} \mathrm{de}$ saliva de los participantes del estudio. La muestra biológica se colectó mediante el kit ORAGENE (DNA Genotek, Canadá), que permitió conservar la muestra hasta su procesamiento para aislar el material genético. La extracción del ADN genómico se realizó siguiendo el protocolo recomendado por el fabricante. Una vez extraído el ADN, se almacenó a $-20^{\circ} \mathrm{C}$ hasta su utilización en las reacciones de amplificación.

\section{Genotipificación}

La genotipificación de los polimorfismos 5HTTLPR y uMAOA, se realizó mediante reacción en cadena de la polimerasa (PCR) con parejas de partidores específicos en un volumen final de reacción de $20 \mu \mathrm{L}$. Ambas reacciones se llevaron a cabo en un termociclador (G-Storm, London, Reino Unido) y los templados fueron separados por electroforesis en geles de agarosa a 2\% (Amresco, Ohio, USA) con buffer de corrida TAE 1X. Los productos de PCR fueron visualizados con tinción de bromuro de etidio, bajo luz ultravioleta. El polimorfismo 5-HTTLPR fue genotipificado en todas las muestras $(\mathrm{n}=1.223)$. La amplificación de ADN genómico se realizó utilizando $50 \mathrm{ng}$ de ADN, 0,25 mM de cada partidor (Sentido: 5-'GGC GTT GCC GCT CTG AAT GCC-3`y Antisentido: 5`CAG GGG AGA TCC TGG GAG AGG T-3`), $200 \mathrm{mM}$ de cada dNTP, 1,5 mM de $\mathrm{MgCl}_{2}$ y 0,3 unidades de Taq polimerasa. Las muestras se amplificaron bajo las siguientes condiciones: ciclo inicial de 8 min a $95^{\circ} \mathrm{C}, 35$ ciclos de: 30 seg a $95^{\circ} \mathrm{C}$, 30 seg a $62^{\circ} \mathrm{C}, 30$ seg a $72^{\circ} \mathrm{C}$ y una extensión final de $1 \mathrm{~min}$ a $72^{\circ} \mathrm{C}$. La PCR para el genotipado de
uMAOA en se realizó con 50 ng de ADN, 1,5 mM $\mathrm{MgCl}_{2}, 200 \mu \mathrm{M}$ de cada dNTP, 10 pmol de cada partidor (Sentido: 5'-ACA GCC TCG CCG TGG AGA AG-3' y Antisentido: 5'-GAA CGG ACG CTC CAT TCG GA-3'), y 1U de Taq polimerasa (Go Taq Green Master Mix, Promega , USA). Las condiciones de amplificación fueron: un ciclo de 5 min a $94^{\circ} \mathrm{C}, 35$ ciclos de $30 \mathrm{seg}$ a $94^{\circ} \mathrm{C}, 30 \mathrm{seg}$ a $55^{\circ} \mathrm{C}$ y 30 seg a $72^{\circ} \mathrm{C}$, con una extensión final a $72^{\circ} \mathrm{C}$ por $5 \mathrm{~min}$.

\section{Variables}

Se recogió información sobre siete factores de riesgo psicosociales y tres variables clínicas (TD, TEPT, TAG), además de polimorfismos en dos genes asociados a depresión para llevar a cabo un modelo de regresión logística binaria.

La principal medida de resultado fue la incidencia de depresión mayor, según criterio DSMIV, lo que se evaluó usando la sección correspondiente del Composite International Diagnostic Interview (CIDI) ${ }^{22,23}$.

Los factores psicosociales seleccionados fueron variables sugeridas por la literatura e incluidas en el estudio PREDICT ${ }^{24}$; se recogieron usando cuestionarios aplicados a los sujetos en entrevistas individuales, cara a cara, donde también se aplicó el CIDI. Los factores considerados fueron edad, sexo, nivel educacional, satisfacción con las condiciones de vida, satisfacción con la relación de pareja, antecedentes de maltrato psicológico. La ocurrencia de eventos vitales se evaluó con la List of Threatening Life Experiences Questionnaire ${ }^{25}$, que fue traducida y adaptada por Motrico y cols. para ser usado en la población consultante en atención primaria ${ }^{26}$. Además, desde la perspectiva clínica, se incluyó el antecedente de depresión en la vida (CIDI), y la auto-percepción de salud física y mental (Short Form 12) ${ }^{27}$.

\section{Análisis estadístico}

El análisis de los datos se realizó empleando una regresión logística binaria. La variable dependiente del modelo fue la presencia del diagnóstico de depresión. En la evaluación de los genotipos para 5HTTLPR se consideró éste como un loci bialélico definido por el tamaño de los fragmentos obtenidos para el gen SLC6A4, alelo corto y largo ( $s$ y $l$, respectivamente). Los alelos de MAOA-uVNTR fueron categorizados de acuerdo a la funcionalidad transcripcional, con la variante 
Tabla 1. Características sociodemográficas y genotípicas de los participantes

\begin{tabular}{|c|c|}
\hline & $\%$ \\
\hline $\begin{array}{l}\text { Depresión } \\
\text { No } \\
\text { Sí }\end{array}$ & $\begin{array}{r}92,6 \\
7,4\end{array}$ \\
\hline $\begin{array}{l}\text { Género } \\
\text { Masculino } \\
\text { Femenino } \\
\text { Edad (media) }\end{array}$ & $\begin{array}{l}25,7 \\
74,3 \\
45,8\end{array}$ \\
\hline $\begin{array}{l}\text { Escolaridad } \\
\text { Sin escolaridad } \\
\text { Ed. Básica } \\
\text { Ed. Media } \\
\text { Ed. Técnica } \\
\text { Ed. Superior }\end{array}$ & $\begin{array}{r}4,1 \\
45,1 \\
42,1 \\
5,8 \\
2,9\end{array}$ \\
\hline $\begin{array}{l}\text { Ocupación } \\
\text { Trabajando } \\
\text { Cesante } \\
\text { Jubilado/Incapacitado } \\
\text { Dueña de casa } \\
\text { Estudiando }\end{array}$ & $\begin{array}{r}28,9 \\
10,0 \\
11,0 \\
42,0 \\
8,1\end{array}$ \\
\hline $\begin{array}{l}\text { Pareja } \\
\text { No } \\
\text { Sí }\end{array}$ & $\begin{array}{l}43,1 \\
56,9\end{array}$ \\
\hline $\begin{array}{l}\text { Maltrato físico } \\
\text { Nunca } \\
\text { A veces } \\
\text { A menudo }\end{array}$ & $\begin{array}{l}64,3 \\
20,5 \\
15,2\end{array}$ \\
\hline $\begin{array}{l}\text { Maltrato psicológico } \\
\text { Nunca } \\
\text { A veces } \\
\text { A menudo }\end{array}$ & $\begin{array}{l}71,5 \\
14,4 \\
14,1\end{array}$ \\
\hline $\begin{array}{l}\text { Abuso sexual } \\
\text { No } \\
\text { Sí }\end{array}$ & $\begin{array}{r}92,2 \\
7,8\end{array}$ \\
\hline $\begin{array}{l}\text { Acontecimientos importantes } \\
\text { Sin eventos } \\
1 \text { evento } \\
2 \text { eventos } \\
3 \text { o más eventos }\end{array}$ & $\begin{array}{l}35,3 \\
28,0 \\
15,7 \\
21,0\end{array}$ \\
\hline $\begin{array}{l}\text { TEPT } 12 \text { meses } \\
\text { No } \\
\text { Sí }\end{array}$ & $\begin{array}{r}96,9 \\
3,1\end{array}$ \\
\hline $\begin{array}{l}\text { TAG } 12 \text { meses } \\
\text { No } \\
\text { Sí }\end{array}$ & $\begin{array}{r}98,9 \\
1,1\end{array}$ \\
\hline $\begin{array}{l}\text { Gen } 5 H T T \text { (5HTTLPR) } \\
\text { II } \\
\text { Is } \\
\text { ss }\end{array}$ & $\begin{array}{l}17,4 \\
47,2 \\
35,4\end{array}$ \\
\hline $\begin{array}{l}\text { Gen MAOA (UMAOA) } \\
\text { Otros } \\
3 / 3\end{array}$ & $\begin{array}{l}82,2 \\
17,8\end{array}$ \\
\hline
\end{tabular}

de 3 repeticiones definida como de baja actividad (codificado 0$)$ y alelos largos $(3,5,4$ y 5 repeticiones) definidos como de alta actividad (codificados 1). Todos los análisis se realizaron empleando el programa Stata 12.1.

\section{Resultados}

\section{Muestra y frecuencias}

Las características sociodemográficas y las variables analizadas se presentan en la Tabla 1. En su mayoría fueron mujeres $74,7 \%$ (914), los hombres representaron 25,7\% (314); el nivel de escolaridad más frecuentemente alcanzado fue educación básica con 45,2\% (553). El 28,9\% (353) de la muestra se encontraba trabajando, $42 \%$ (514) eran dueñas de casa, 10\% (122) se encontraba desempleado y $11 \%$ (135) jubilado o incapacitado, mientras que $8,1 \%$ (99) eran estudiantes. La presencia de maltrato físico, independiente de la frecuencia con que se daba, alcanzó a 35,7\% (436) de la muestra, así también en maltrato psicológico se encontró en $28,5 \%$ (349), mientras que abuso sexual fue reportado por 7,8\% (95). La presencia de acontecimientos vitales importantes totales mostró una frecuencia de 64,7 \% (791). El TPET fue descrito en 3,1\% (38) y el TAG sólo en 1,1\% (13). Las frecuencias para los tres genotipos del polimorfismo 5-HTTLPR fueron los siguientes: largo/largo (1/l): 17,4\% (213); largo/corto (1/s): 47,2\% (577); y corto/corto (s/s): $35,4 \%$ (433). En tanto para la variante uMAOA la frecuencia para el genotipo $3 / 3$ fue de $17,8 \%$ (218).

\section{Asociación entre variables y depresión}

Los resultados de los análisis de la regresión logística para establecer asociación entre depresión y la presencia o ausencia de variables psicosociales y genéticas se presentan en la Tabla 2. De las variables evaluadas como edad, nivel de escolaridad, ocupación, antecedentes de maltrato físico, trastorno estrés post traumático, trastorno de ansiedad generalizado y los genotipos ll, ls y ss para 5HTTLPR y $3 / 3$ para uMAOA no se detectó una asociación significativa con depresión en la población evaluada. A diferencia de esto, hay concordancia que el género femenino puede presentar el doble de riesgo de presentar depresión, mientras que las variables psicosociales 
Tabla 2. Análisis de regresión logística para establecer eventuales asociaciones entre depresión y variables psico-socio-genéticas

\begin{tabular}{|c|c|c|c|}
\hline & OR & IC95\% & Wald-F test \\
\hline $\begin{array}{l}\text { Edad } \\
\text { Femenino }\end{array}$ & $\begin{array}{l}1,000 \\
1,984^{* *}\end{array}$ & $\begin{array}{l}(0,988-1,012) \\
(1,221-3,226)\end{array}$ & \\
\hline $\begin{array}{l}\text { Educación } \\
\text { Superior } \\
\text { Secundaria } \\
\text { Primaria/Sin educación } \\
\text { Otro }\end{array}$ & $\begin{array}{l}\text { Referencia } \\
0,873 \\
1,364 \\
1,354\end{array}$ & $\begin{array}{l}(0,199-3,834) \\
(0,329-5,661) \\
(0,268-6,828)\end{array}$ & $11,06^{* * *}$ \\
\hline $\begin{array}{l}\text { Satisfacción con las condiciones de vida } \\
\text { Satisfecho } \\
\text { Ni satisfecho, ni insatisfecho } \\
\text { Insatisfecho }\end{array}$ & $\begin{array}{l}\text { Referencia } \\
0,787 \\
1,670 * * *\end{array}$ & $\begin{array}{l}(0,508-1,218) \\
(1,226-2,275)\end{array}$ & $5,98^{* *}$ \\
\hline $\begin{array}{l}\text { Depresión-Vida } \\
\text { Salud física } \\
\text { Salud mental }\end{array}$ & $\begin{array}{l}2,476 * * * \\
0,963 * * * \\
0,963 * * *\end{array}$ & $\begin{array}{l}(1,901-3,224) \\
(0,942-0,985) \\
(0,952-0,975)\end{array}$ & \\
\hline $\begin{array}{l}\text { Satisfacción con la relación de pareja } \\
\text { Satisfecho } \\
\text { Insatisfecho } \\
\text { Sin pareja/esposo(a) }\end{array}$ & $\begin{array}{c}\text { Referencia } \\
1,264 \\
0,661^{*}\end{array}$ & $\begin{array}{l}(0,971-1,645) \\
(0,456-0,958)\end{array}$ & $4,94^{* *}$ \\
\hline $\begin{array}{l}\text { Maltrato psicológico } \\
\text { Nunca } \\
\text { A veces } \\
\text { A menudo }\end{array}$ & $\begin{array}{c}\text { Referencia } \\
2,186^{*} \\
1,809\end{array}$ & & $6,06^{*}$ \\
\hline $\begin{array}{l}\text { Acontecimientos Importantes } \\
\text { Sin eventos } \\
1 \text { evento } \\
2 \text { eventos } \\
3 \text { o más eventos }\end{array}$ & $\begin{array}{l}1,000 \\
1,457 \\
2,026^{*} \\
2,454^{\star}\end{array}$ & & $8,86^{*}$ \\
\hline $\begin{array}{l}\text { Gen } 5 H T T(5 H T T L P R) \\
\text { Is } \\
\text { II } \\
\text { ss }\end{array}$ & $\begin{array}{c}\text { Referencia } \\
0,902 \\
1,056\end{array}$ & $\begin{array}{l}(0,567-1,436) \\
(0,737-1,513)\end{array}$ & 0,26 \\
\hline $\begin{array}{l}\text { Gen MAOA (UMAO) } \\
\text { Otros } \\
3 / 3\end{array}$ & $\begin{array}{c}\text { Referencia } \\
1,002\end{array}$ & $(0,718-1,400)$ & \\
\hline Constante & 1,409 & $(0,141-14,064)$ & \\
\hline
\end{tabular}

${ }^{*} p<0,05 ;{ }^{* *} p<0,01 ; * * * p<0,001$.

como: maltrato psicológico (OR: 2,186 \pm 0,711, $\mathrm{p}<0,05)$, la presencia de dos o más eventos vitales importantes (OR: 2,026 \pm 0,711, OR: 2,454 \pm $0,780, \mathrm{p}<0,05)$ y el grado de insatisfacción con las condiciones de vida (OR: 1,670, IC: 1,2262,275 ) mostraron asociación significativa con depresión.

\section{Discusión}

El presente estudio no detectó asociación entre los genotipos de riesgo de los polimorfismos 5 HTTLPR (s/s), uMAOA (3/3) y la presencia de depresión en la cohorte evaluada, por tanto, no constituyen marcadores de riesgo para este 
trastorno en esta población. A diferencia de este resultado, las variables psicosociales evaluadas: maltrato psicológico, la presencia de dos o más eventos vitales y el grado de insatisfacción con la condiciones de vida, sí están asociados a la patología y pertenecer al género femenino aumenta al doble el riesgo de presentar depresión.

Este estudio se realizó en una muestra representativa de la población consultante en atención primaria, lo que permite afirmar que sus resultados son en buena medida generalizables. Por otra parte, dado que el sistema de salud chileno es altamente estructurado y alcanza altos niveles de cobertura, permite una razonable aproximación a las características de la población general. El diseño y la técnica de muestreo, así como el uso de instrumentos diagnósticos estructurados, permiten un adecuado control de sesgos y aseguran confiabilidad de los resultados. Sin embargo, la relativa alta tasa de pérdidas hace necesario analizar los resultados cuidadosamente.

El estudio PREDICT-GENE ${ }^{28}$, que describió asociación entre algunos de estos factores psicosociales y depresión, coincide parcialmente con nuestros resultados. La imposibilidad de reproducir los resultados genéticos de las variantes en estudio, descritas en distintas poblaciones ${ }^{28-33}$, sugiere que el componente étnico debería ser un factor a considerar en la selección de genes candidatos. Actualmente, la búsqueda de genes candidatos se basa en los antecedentes de la posible implicación de éstos en los sistemas biológicos afectados en depresión, en base a las frecuencias de los polimorfismos descritos en poblaciones caucásicas.

Nuestros resultados negativos coinciden con otras investigaciones, que no detectaron asociación entre depresión y la variante 5HTTLPR del transportador de serotonina $a^{34-36}$ o la variante uMAOA de la monoamino oxidasa ${ }^{37}$. Esto puede deberse a las diferencias metodológicas existentes entre estudios, tales como el diseño, la población analizada, el propio diagnóstico de depresión mayor, o incluso la falta de poder estadístico debido al pequeño tamaño de la muestra. Sin embargo, Mendlewicz y cols ${ }^{28}$ realizaron un estudio multicéntrico de asociación bajo estrictos controles, con un diseño caso-control en una amplia muestra de sujetos europeos $(\mathrm{n}=1.111)$; el objetivo fue evaluar el efecto del polimorfimo 5HTTLPR en los fenotipos de trastornos afectivos: depresión mayor y depresión bipolar y, a pesar de parear a los participantes por zona geografica, los autores no detectaron asociación entre los fenotipos y las variantes de estudio ${ }^{34}$. Por otra parte, encontramos ejemplos de asociaciones discretas, pero positivas, entre la presencia del alelo $S$ para el 5HTTLPR y la presencia de un grupo de síntomas del trastorno depresivo mayor en población japonesa ${ }^{38}$. Particularmente, en nuestro trabajo no se realizó la reclasificación de los sujetos que portan la variante I para el 5HTTLPR de acuerdo al polimorfismo de nucleótido único (SNP, A/G), presente en la región polimórfica del transportador (SERT), lo que podría constituir probablemente una limitación del estudio, ya que los sujetos con la variante $I_{G}$ se comportan fenotípicamente como portadores del genotipo ss. Por otra parte, tal como se señaló anteriormente, nuestro componente amerindio, el cual no fue evaluado, podría dar cuenta de la baja reproducibilidad de los resultados obtenidos en relación a estos genes como marcadores de riesgo genético y depresión en población española.

Luego de analizar las variables psicosociales y genéticas, este estudio concluye que no existe asociación entre depresión y las variantes 5HTTLPR y uMAOA en los genes SLC6V4 y MAOA, respectivamente. Sin embargo, se debe tener en cuenta que la variablidad genética en genes que confieren un riesgo de efecto menor se deben abordar hoy en día con una perspectiva epigenética, ya que la interacción entre los genotipos y el medio ambiente modulan su expresión fenotipica y por tanto, tambien la vulnerabilidad a depresión.

En conclusión, el presente estudio, el primero en el país en incorporar factores psicosociales y genéticos que se relacionan con la aparición de depresión a través de un diseño prospectivo, no permitió replicar los resultados que asocian las variantes 5HTTLPR y uMAOA como factores de riesgo a depresión, pero confirma que el maltrato psicológico y la presencia de dos o más eventos vitales son factores de riesgo para presentar una patología depresiva. Las principales limitaciones son el pequeño número de genes estudiados y una relativa alta tasa de pérdidas.

En futuros estudios se deberían incluir un mayor número de genes que permita identificar las características genéticas de riesgo que esta población presenta y evaluar intervenciones preventivas selectivas sobre las personas con mayor riesgo. 


\section{Referencias}

1. Sullivan PF, Neale MC, Kendler KS. Genetic epidemiology of major depression: review and meta-analysis. Am J Psychiatry 2000; 157 (10): 1552-62.

2. Flint J, Kendler KS. The Genetics of Major Depression. Neuron 2014; 81 (3): 484-503.

3. Wender PH, Kety SS, Rosenthal D, Schulsinger F, Ortmann J, Lunde I. Psychiatric disorders in the biological and adoptive families of adopted individuals with affective disorders. Arch Gen Psychiatry 1986; 43 (10): 923-9.

4. Levinson DF. The genetics of depression: a review. Biol Psychiatry 2006; 60 (2): 84-92.

5. Ebmeier KP, Donaghey C, Steele JD. Recent developments and current controversies in depression. Lancet 2006; 367 (9505): 153-67.

6. Cervilla JA, Rivera M, Molina E, Torres-González F, Bellon JA, Moreno B, et al. The 5-HTTLPR s/s genotype at the serotonin transporter gene (SLC6A4) increases the risk for depression in a large cohort of primary care attendees: the PREDICT-gene study. Am J Med Genet B Neuropsychiatr Genet 2006; 141B (8): 912-7.

7. Gutiérrez B, Arranz MJ, Collier DA, Valles V, Guillamat $\mathrm{R}$, Bertranpetit J, et al. Serotonin transporter gene and risk for bipolar affective disorder: an association study in Spanish population. Biol Psychiatry 1998; 43 (11): 843-7.

8. Lesch KP, Bengel D, Heils A, Sabol SZ, Greenberg BD, Petri S, et al. Association of anxiety-related traits with a polymorphism in the serotonin transporter gene regulatory region. Science 1996; 274 (5292): 1527-31.

9. López-León S, Janssens AC, González-Zuloeta Ladd AM, Del-Favero J, Claes SJ, Oostra BA, et al. Meta-analyses of genetic studies on major depressive disorder. Mol Psychiatry 2008; 13 (8): 772-85.

10. Heils A, Teufel A, Petri S, Stober G, Riederer P, Bengel $\mathrm{D}$, et al. Allelic variation of human serotonin transporter gene expression. J Neurochem 1996; 66 (6): 2621-4.

11. Kraft JB, Slager SL, McGrath PJ, Hamilton SP. Sequence analysis of the serotonin transporter and associations with antidepressant response. Biol Psychiatry 2005; 58 (5): 374-81.

12. Nakamura M, Ueno S, Sano A, Tanabe H. The human serotonin transporter gene linked polymorphism (5-HTTLPR) shows ten novel allelic variants. Mol Psychiatry 2000; 5 (1): 32-8.

13. Arias B, Catalan R, Gasto C, Gutiérrez B, Fananas L. 5-HTTLPR polymorphism of the serotonin transporter gene predicts non-remission in major depression patients treated with citalopram in a 12 -weeks follow up study. J Clin Psychopharmacol 2003; 23 (6): 563-7.
14. Serretti A, Zanardi R, Rossini D, Cusin C, Lilli R, Smeraldi E. Influence of tryptophan hydroxylase and serotonin transporter genes on fluvoxamine antidepressant activity. Mol Psychiatry 2001; 6 (5): 586-92.

15. Zanardi R, Benedetti F, Di Bella D, Catalano M, Smeraldi E. Efficacy of paroxetine in depression is influenced by a functional polymorphism within the promoter of the serotonin transporter gene. J Clin Psychopharmacol 2000; 20 (1): 105-7.

16. Lim JE, Papp A, Pinsonneault J, Sadee W, Saffen D. Allelic expression of serotonin transporter (SERT) mRNA in human pons: lack of correlation with the polymorphism SERTLPR. Mol Psychiatry 2006; 11 (7): 649-62.

17. Parsey RV, Hastings RS, Oquendo MA, Huang YY, Simpson N, Arcement J, et al. Lower serotonin transporter binding potential in the human brain during major depressive episodes. Am J Psychiatry 2006; 163 (1): 52-8.

18. Sabol SZ, Hu S, Hamer D. A functional polymorphism in the monoamine oxidase A gene promoter. Hum Genet 1998; 103 (3): 273-9.

19. Deckert J, Catalano M, Syagailo YV, Bosi M, Okladnova $\mathrm{O}$, Di Bella D, et al. Excess of high activity monoamine oxidase A gene promoter alleles in female patients with panic disorder. Hum Mol Genet 1999; 8 (4): 621-4.

20. Du L, Faludi G, Palkovits M, Sotonyi P, Bakish D, Hrdina PD. High activity-related allele of MAO-A gene associated with depressed suicide in males. Neuroreport 2002; 13 (9): 1195-8.

21. Rivera M, Gutiérrez B, Molina E, Torres-González F, Bellon JA, Moreno-Kustner B, et al. High-activity variants of the uMAOA polymorphism increase the risk for depression in a large primary care sample. Am J Med Genet B Neuropsychiatr Genet 2009; 150B (3): 395-402.

22. Organization $\mathrm{WH}$. Composite International Diagnostic Interview (CIDI). Version 2.1. Geneva, Switzerland. WHO. 1997.

23. Robins LN, Wing J, Wittchen HU, Helzer JE, Babor TF, Burke J, et al. The Composite International Diagnostic Interview. An epidemiologic Instrument suitable for use in conjunction with different diagnostic systems and in different cultures. Arch Gen Psychiatry 1988; 45 (12): 1069-77.

24. King M, Weich S, Torres-González F, Svab I, Maaroos $\mathrm{HI}$, Neeleman J, et al. Prediction of depression in European general practice attendees: the PREDICT study. BMC Public Health 2006; 6: 6.

25. Brugha T, Bebbington P, Tennant C, Hurry J. The List of Threatening Experiences: a subset of 12 life event categories with considerable long-term contextual threat. Psychol Med 1985; 15 (1): 189-94. 
26. Motrico E, Moreno-Kustner B, de Dios Luna J, Torres-González F, King M, Nazareth I, et al. Psychometric properties of the List of Threatening Experiences-LTE and its association with psychosocial factors and mental disorders according to different scoring methods. J Affect Disord 2013; 150 (3): 931-40.

27. Jenkinson C, Layte R, Jenkinson D, Lawrence K, Petersen S, Paice C, et al. A shorter form health survey: can the SF-12 replicate results from the SF-36 in longitudinal studies? J Public Health Med 1997; 19 (2): 179-86.

28. Cervilla JA, Molina E, Rivera M, Torres-González F, Bellon JA, Moreno B, et al. The risk for depression conferred by stressful life events is modified by variation at the serotonin transporter 5HTTLPR genotype: evidence from the Spanish PREDICT-Gene cohort. Mol Psychiatry 2007; 12 (8): 748-55.

29. Furlong RA, Ho L, Walsh C, Rubinsztein JS, Jain S, Paykel ES, et al. Analysis and meta-analysis of two serotonin transporter gene polymorphisms in bipolar and unipolar affective disorders. Am J Med Genet 1998; 81 (1): 58-63.

30. Gutiérrez B, Pintor L, Gasto C, Rosa A, Bertranpetit J, Vieta E, et al. Variability in the serotonin transporter gene and increased risk for major depression with melancholia. Hum Genet 1998; 103 (3): 319-22.

31. Kunugi H, Hattori M, Kato T, Tatsumi M, Sakai T, Sasaki T, et al. Serotonin transporter gene polymorphisms: ethnic difference and possible association with bipolar affective disorder. Mol Psychiatry 1997; 2 (6): 457-62.
32. Lotrich FE, Pollock BG. Meta-analysis of serotonin transporter polymorphisms and affective disorders. Psychiatr Genet 2004; 14 (3): 121-9.

33. Xu J, Cheng YQ, Chen B, Bai R, Li S, Xu XF, et al. Depression in systemic lupus erythematosus patients is associated with link-polymorphism but not methylation status of the 5HTT promoter region. Lupus 2013; 22 (10): 1001-10.

34. Mendlewicz J, Massat I, Souery D, Del-Favero J, Oruc L, Nothen MM, et al. Serotonin transporter 5HTTLPR polymorphism and affective disorders: no evidence of association in a large European multicenter study. Eur J Hum Genet 2004; 12 (5): 377-82.

35. Minov C, Baghai TC, Schule C, Zwanzger P, Schwarz $\mathrm{MJ}$, Zill P, et al. Serotonin-2A-receptor and -transporter polymorphisms: lack of association in patients with major depression. Neurosci Lett 2001; 303 (2): 119-22.

36. Ohara K, Nagai M, Tsukamoto T, Tani K, Suzuki Y, Ohara K. Functional polymorphism in the serotonin transporter promoter at the SLC6A4 locus and mood disorders. Biol Psychiatry 1998; 44 (7): 550-4.

37. Syagailo YV, Stober G, Grassle M, Reimer E, Knapp M, Jungkunz G, et al. Association analysis of the functional monoamine oxidase A gene promoter polymorphism in psychiatric disorders. Am J Med Genet 2001; 105 (2): 168-71.

38. Kamata M, Suzuki A, Yoshida K, Takahashi H, Higuchi $\mathrm{H}$, Otani K. Genetic polymorphisms in the serotonergic system and symptom clusters of major depressive disorder. J Affect Disord 2011; 135 (1-3): 374-6. 